\title{
The influence of scar on the spatio-temporal relationship between electrical and mechanical activation in heart failure patients
}

Citation for published version (APA):

Maffessanti, F., Jadczyk, T., Kurzelowski, R., Regoli, F., Caputo, M. L., Conte, G., Golba, K. S., Biernat, J., Wilczek, J., Dabrowska, M., Pezzuto, S., Moccetti, T., Krause, R., Wojakowski, W., Prinzen, F. W., \& Auricchio, A. (2020). The influence of scar on the spatio-temporal relationship between electrical and mechanical activation in heart failure patients. EP Europace, 22(5), 777-786.

https://doi.org/10.1093/europace/euz346

Document status and date:

Published: 01/05/2020

DOI:

10.1093/europace/euz346

Document Version:

Publisher's PDF, also known as Version of record

Document license:

Taverne

Please check the document version of this publication:

- A submitted manuscript is the version of the article upon submission and before peer-review. There can be important differences between the submitted version and the official published version of record.

People interested in the research are advised to contact the author for the final version of the publication, or visit the DOI to the publisher's website.

- The final author version and the galley proof are versions of the publication after peer review.

- The final published version features the final layout of the paper including the volume, issue and page numbers.

Link to publication

\footnotetext{
General rights rights.

- You may freely distribute the URL identifying the publication in the public portal. please follow below link for the End User Agreement:

www.umlib.nl/taverne-license

Take down policy

If you believe that this document breaches copyright please contact us at:

repository@maastrichtuniversity.nl

providing details and we will investigate your claim.
}

Copyright and moral rights for the publications made accessible in the public portal are retained by the authors and/or other copyright owners and it is a condition of accessing publications that users recognise and abide by the legal requirements associated with these

- Users may download and print one copy of any publication from the public portal for the purpose of private study or research.

- You may not further distribute the material or use it for any profit-making activity or commercial gain

If the publication is distributed under the terms of Article 25fa of the Dutch Copyright Act, indicated by the "Taverne" license above, 


\title{
The influence of scar on the spatio-temporal relationship between electrical and mechanical activation in heart failure patients
}

\author{
Francesco Maffessanti (i) ${ }^{1 *}$, Tomasz Jadczyk ${ }^{2,3}$, Radosław Kurzelowski ${ }^{2}$, \\ François Regoli ${ }^{4}$, Maria Luce Caputo ${ }^{4}$, Giulio Conte ${ }^{4}$, Krzysztof S. Gołba ${ }^{5}$, \\ Jolanta Biernat ${ }^{5}$, Jacek Wilczek ${ }^{5}$, Magdalena Dabrowska ${ }^{5}$, Simone Pezzuto', \\ Tiziano Moccetti ${ }^{4}$, Rolf Krause ${ }^{1}$, Wojciech Wojakowski ${ }^{2}$, Frits W. Prinzen ${ }^{6}$, and \\ Angelo Auricchio ${ }^{1,4}$
} ${ }^{1}$ Center for Computational Medicine in Cardiology, Università della Svizzera italiana, Via G. Buffi $13, \mathrm{CH}-6900$ Lugano, Switzerland; ${ }^{2}$ Division of Cardiology and Structural Heart
Diseases, Medical University of Silesia, Katowice, Poland; Interventional Cardiac Electrophysiology Group, International Clinical Research Center, St. Anne's University Hospital
Brno, Czech Republic; ${ }^{4}$ Division of Cardiology, Fondazione Cardiocentro Ticino, Lugano, Switzerland; ${ }^{5}$ Department of Electrocardiology and Heart Failure, Medical University of
Silesia, Katowice, Poland; and ${ }^{6}$ Department of Physiology, CARIM, Maastricht University, Maastricht, The Netherlands

Received 19 August 2019; editorial decision 1 December 2019; accepted 5 December 2019; online publish-ahead-of-print 14 January 2020

\begin{abstract}
Aims The aim of this study was to determine the relationship between electrical and mechanical activation in heart failure (HF) patients and whether electromechanical coupling is affected by scar.

Methods

and results

Seventy HF patients referred for cardiac resynchronization therapy or biological therapy underwent endocardial anatomo-electromechanical mapping (AEMM) and delayed-enhancement magnetic resonance (CMR) scans. Area strain and activation times were derived from AEMM data, allowing to correlate mechanical and electrical activation in time and space with unprecedented accuracy. Special attention was paid to the effect of presence of CMRevidenced scar. Patients were divided into a scar $(n=43)$ and a non-scar group $(n-27)$. Correlation between time of electrical and mechanical activation was stronger in the non-scar compared to the scar group $[R=0.84(0.72-$ $0.89)$ vs. $0.74(0.52-0.88)$, respectively; $P=0.01]$. The overlap between latest electrical and mechanical activation areas was larger in the absence than in presence of scar [72\% (54-81) vs. 56\% (36-73), respectively; $P=0.02$ ], with smaller distance between the centroids of the two regions [10.7 (4.9-17.4) vs. 20.3 (6.9-29.4) \% of left ventricular radius, $P=0.02]$.
\end{abstract}

\section{Conclusion}

Keywords
Scar decreases the association between electrical and mechanical activation, even when scar is remote from late activated regions.

Heart failure - Cardiac resynchronization therapy - Cardiac magnetic resonance - Activation • Left bundle branch block - Scar

\section{Introduction}

Cardiac resynchronization therapy (CRT) is a valuable adjunct therapy in patients with heart failure (HF) and conduction delay. Several studies have shown that pacing the left ventricle (LV) in a region of late activation results in larger LV reverse remodelling, better quality of life, and improved survival of CRT patients. ${ }^{1-4}$ In clinical practice, electrophysiologists either target a pre-procedurally and noninvasively determined area with late peak shortening, ${ }^{5}$ or the location at the LV wall with the longest electrical delay as compared to the right ventricular (RV) lead (RV-LV interval) or the $\mathrm{Q}$ wave on surface electrocardiogram (ECG; Q-LV interval), ${ }^{6}$ both of which are

* Corresponding author. Tel: +41 (0)58 666 4812; fax: +41 (0)58 666 4536. E-mail address: maffef@usi.ch

Published on behalf of the European Society of Cardiology. All rights reserved. (c) The Author(s) 2020. For permissions, please email: journals.permissions@oup.com. 


\section{What's new?}

- Heart failure patients show a large variability in terms of electromechanical (EM) coupling, particularly in the presence of scar.

- Latest mechanical activation is less likely to occur in the lateral left ventricular wall than latest electrical activation, especially in the presence of scar.

- The effect of scar appears independent of the distance between scar and latest activated regions, suggesting a remote influence on EM coupling.

determined invasively and during the implant procedure. Clearly, determination of the best $L V$ pacing location prior to the implant procedure in a non-invasive manner is to be preferred, but this can only be achieved if late mechanical activation also coincides with late electrical activation.

In a healthy heart, the intact electromechanical (EM) coupling ensures a good agreement of the areas of late mechanical and electrical activations. ${ }^{7}$ Whether this holds true in patients with HF, and different degrees of ventricular conduction delay is less clear, especially in patients with an ischaemic aetiology of HF and presence of myocardial scar. The few studies that have until now investigated the agreement between the area of late electrical and late mechanical activation reported conflicting results. ${ }^{8-10}$ Of note, all these studies suffered from the limitation that the methods used for measuring electrical and mechanical activations were different, thus requiring complex image integration and were performed sequentially on different heart beats and at different times. These limitations can be overcome by anatomo-electromechanical mapping (AEMM), a technique recently developed and validated by our group. ${ }^{11,12}$ Here, the local electrical activation is determined by a mapping catheter, while the catheter motion is used to assess mechanical activation on the same beat and location. ${ }^{12}$

The aims of this study were (i) to identify the most common areas of latest electrical and latest mechanical activation and their spatial match in a group of HF patients, most of them having a wide QRS complex; and (ii) to assess to what extent scar affects EM coupling in these hearts and the spatial match between regions of late electrical and mechanical activation.

\section{Methods}

\section{Population and study design}

Seventy-five patients with moderate-to-severe HF were prospectively studied at two centres, Cardiocentro Ticino $(n=38$, Lugano, Switzerland) and the Medical University of Silesia $(n=37$, Katowice, Poland). All patients were either candidate for implantation of a CRT device $(n=66)$ or for intramyocardial biological therapy $(n=9)$, being in stable drug therapy at the time of enrolment, with a LV ejection fraction $<35 \%$, NYHA (New York Heart Association) Class II-IV, and a QRS duration $>120 \mathrm{~ms}$. Each patient underwent standard 12-lead ECG, cardiovascular magnetic resonance (CMR) imaging for scar evaluation, and an AEMM study. The study was approved by the Institutional Review Board of each centre, and oral and written informed consent was obtained from each patient before the enrolment.

\section{Electroanatomic mapping}

Electromechanical mapping of the LV was performed using the NOGAXP system (Biologic Delivery Systems, Division of Biosense Webster a Johnson \& Johnson Company) and a conventional 7-Fr deflectable-tip mapping catheter (NAVI-STAR, Biosense Webster). The system and the navigation method have been described in detail previously. ${ }^{11,12}$ Intracardiac unipolar (UEG) and bipolar electrograms, and the spatial position of the catheter were recorded at $\sim 200 \mathrm{LV}$ endocardial locations. UEGs and the corresponding position of the tip of the mapping catheter were simultaneously recorded at $1 \mathrm{KHz}$ and $100 \mathrm{~Hz}$, respectively, at different locations spreading the whole endocardium. The sequentially recorded UEGs and position data were temporally aligned using the simultaneously recorded 12-lead surface ECG.

\section{Post-processing}

Data sets from EM mapping were processed in MATLAB (Mathworks, USA) to obtain AEMM measurements (Supplementary material online, Figure S1). First, a set of stability criteria was applied to remove all those points showing non-physiological large spatial gradients or visually belonging to the papillary muscles. Local time of depolarization (TD) was identified as the time of the maximum negative slope of UEG $(\max (-d U E G / d t))$ in a time window including the entire QRS complex. The measurement of endocardial local area strain from the three-dimensional displacement field of the catheter tips has been previously described in detail. ${ }^{12}$ Time-to-peak shortening (TPS) for each region was identified as the time corresponding to the first peak of the local area strain curve. The earliest TD measured in the LV endocardium was used as reference for TD and TPS.

The overlap between the areas of latest electrical and mechanical activation was evaluated using the regions activated after $80 \%$ of the corresponding activation and expressed as percentage of the area of latest electrical activation. Also, the centroids of the latest activated areas were identified, and their distance was expressed as \% of the equivalent radius of the LV.

\section{Cardiovascular magnetic resonance}

Cardiovascular magnetic resonance imaging was performed using a $3 \mathrm{~T}$ scanner (MAGNETOM Skyra, Siemens Healthcare, Germany) in Lugano and a 1.5T scanner in Katowice (SIGNA, GE Medical Systems, USA) equipped with standard torso coil. Short-axis late gadolinium enhancement (LGE) images were obtained 7-12 min after the intravenous bolus injection of gadolinium $(0.2 \mathrm{mmol} / \mathrm{kg}$ body weight). Each short-axis slice was subdivided into the 16 angular sectors, according to the AHA standard and area with LGE automatically segmented with the full-width halfmaximum criterion. Each short-axis slice was subdivided into 32 angular subregions and the local scar burden was expressed as the percent of scar per subregion, normalized by the area of the subregion itself. Significant scar was defined in presence of scar burden $>50 \%$ in at least one angular sector.

\section{Integration of late gadolinium enhancement cardiovascular magnetic resonance with anatomo-electromechanical mapping}

Information from LGE maps was integrated with data obtained from AEMM in a two-step algorithm. Briefly, the centres of mass of LGE and AEMM surfaces were aligned using a three-dimensional translation, then an iterative closest-point approach was used to minimize the Euclidean distance between the electrodes and the endocardial contours traced on CMR images. ${ }^{13}$ 
Table I Patients characteristics

\begin{tabular}{|c|c|c|c|c|}
\hline & Overall & Centre number 1 & Centre number 2 & $P$-value \\
\hline \multicolumn{5}{|l|}{ Demographics } \\
\hline Number of patients & 70 & 34 & 36 & - \\
\hline Male & $52(74)$ & $25(74)$ & $27(75)$ & 1.00 \\
\hline Ischaemic cardiomyopathy & $55(79)$ & $19(56)$ & $36(100)$ & $<0.01$ \\
\hline NYHA (I/II/III/IV) & $0(0) / 33(47) / 36(51) / 1(1)$ & $0(0) / 15(44) / 18(53) / 1(3)$ & $0(0) / 18(50) / 18(50) / 0(0)$ & 0.75 \\
\hline CRT & $62(89)$ & $26(76)$ & $36(100)$ & $<0.01$ \\
\hline Biological therapy & $8(70)$ & $8(24)$ & $0(0)$ & $<0.01$ \\
\hline \multicolumn{5}{|l|}{ ECG characteristics } \\
\hline Sinus rhythm & $58(83)$ & $30(88)$ & $28(78)$ & 0.35 \\
\hline Atrial fibrillation & $12(17)$ & $4(12)$ & $8(22)$ & 0.35 \\
\hline Heart rate (b.p.m.) & $69(60-77)$ & $70(59-83)$ & $68(60-75)$ & 0.48 \\
\hline QRS duration (ms) & $157(143-173)$ & $156(138-178)$ & $159(145-170)$ & 0.23 \\
\hline LBBB & $57(81)$ & $21(62)$ & $36(100)$ & $<0.01$ \\
\hline IVCD & $8(11)$ & $8(24)$ & $0(0)$ & $<0.01$ \\
\hline \multicolumn{5}{|l|}{ CMR LV function } \\
\hline $\mathrm{EF}(\%)$ & $25(21-31)$ & $25(21-32)$ & $24(21-31)$ & 0.39 \\
\hline Mass (g) & $156(137-187)$ & $155(140-175)$ & $160(134-192)$ & 0.42 \\
\hline $\mathrm{EDV}(\mathrm{mL})$ & $257(224-326)$ & $255(216-320)$ & $269(231-328)$ & 0.91 \\
\hline ESV (mL) & $194(156-250)$ & $198(143-240)$ & $194(165-252)$ & 0.80 \\
\hline \multicolumn{5}{|l|}{ Medications } \\
\hline ACE inhibitors & $62(89)$ & 31 (91) & $31(86)$ & 0.71 \\
\hline Diuretics & $60(86)$ & $27(79)$ & $33(92)$ & 0.18 \\
\hline Beta-blockers & $70(100)$ & $34(100)$ & $36(100)$ & 1.00 \\
\hline
\end{tabular}

\section{Statistical analysis}

For each patient, correlation analysis was performed to establish the association between TD and TPS. The evolution of the activated area has been normalized in time for the length of the corresponding interval, electrical or mechanical, and over space with respect to the total endocardial area and expressed as percentage, to allow comparison among patients. The electrical and mechanical activation curves were compared measuring the area between the two curves, expressed as percentage of the maximum area and using Kolmogorov-Smirnov statistic. Significance of the scar vs. non-scar groups comparison was tested using MannWhitney $U$ test. In order to investigate the effect of the threshold used to define significant scar on the results, the analysis has been repeated separately for scar transmurality in the $50-75 \%$ and $>75 \%$ ranges.

All data area expressed as mean $\pm \mathrm{SD}$, median (1st-3rd) quartiles, or counts and percentage values, as appropriate.

\section{Results}

Late gadolinium enhancement imaging was feasible in all but five patients due to claustrophobia and these cases were excluded. Table 1 summarizes the demographic and clinical characteristics of the remaining 70 patients. A transmural scar, i.e. $>50 \%$ of the area in at least one of the 32 sectors in which each slice was subdivided, was found in 43 patients. Scar was either endocardial or transmural; no isolated epicardial scar was observed. Anatomo-electromechanical mapping was possible in all cases (Table 2).

\section{Extent and location of latest activated regions}

Figure 1depicts EM association in two representative patients. In both cases, the electrical activation spread gradually from the anteroseptal towards the endocardial inferolateral region. Optimal EM coupling in Patient $A$ is confirmed by the similar patterns of electrical and mechanical activation (upper panels), similar curves describing progress of activation as a function of LV endocardial area (bottom left) and by the high TD-TPS correlation (bottom right). Patient B (right panel) shows clear discrepancies between the electrical and mechanical maps, with the mechanical activation following an infero-septal to antero-lateral evolution pattern. Of note, the location associated with the latest electrical activation (red marker 2) was not associated with a latest mechanical event (green marker 2 falls in the middle of the green interval). The poorer EM coupling in this patient was further evidenced by slower progression of mechanical than electrical activation along the LV endocardial area (bottom left panel) and the poor TD-TPS correlation (bottom right). This correlation remained poor after exclusion of the regions associated with scar (grey data points). Table 2 shows that strength of the TD-TPS relation was significantly weaker in scarred than in non-scarred hearts. 
Table 2 Summary of all the measurements of electrical and mechanical activation for the entire study group and separately for non-scar and scar patients

\begin{tabular}{|c|c|c|c|c|}
\hline & All patients $(n=70)$ & Non-scar $(n=27)$ & Scar $(n=43)$ & $P$-value \\
\hline Number of endocardial sites $(n)$ & $225(179-254)$ & $203(160-244)$ & $229(198-256)$ & 0.08 \\
\hline TD-TPS correlation (R) & $0.81(0.62-0.88)$ & $0.84(0.72-0.89)$ & $0.74(0.52-0.88)$ & 0.01 \\
\hline Area between $\mathrm{E}$ and $\mathrm{M}$ curves over time (\%) & $11.8(6.5-16.3)$ & $8.9(3.8-13.6)$ & $13.9(7.6-16.9)$ & 0.03 \\
\hline Distance between electrical and mechanical centroids (\%) & $12.8(7.0-28.1)$ & $10.7(4.9-17.4)$ & $20.3(6.9-29.4)$ & 0.02 \\
\hline Overlap (\%) & $64(41-78)$ & $72(54-81)$ & $56(36-73)$ & 0.02 \\
\hline
\end{tabular}

$P$-values refer to non-scar vs. scar and Mann-Whitney $U$ test. Data are expressed as median (1st-3rd) quartiles.

TD, time of depolarization; TPS, time-to-peak shortening.

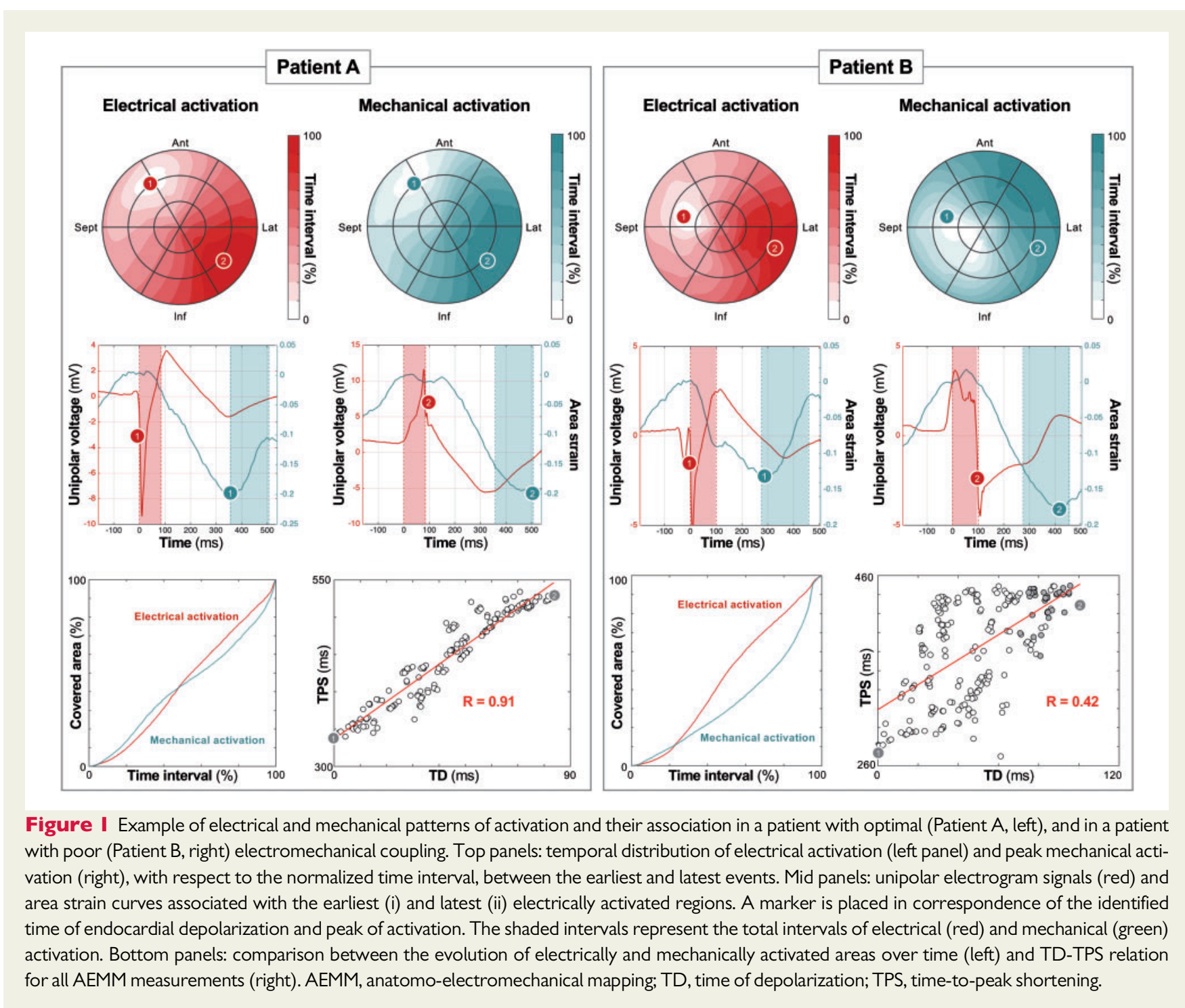

Figure 2 summarizes, for the whole study group, the temporal evolution of the LV area that was electrically (red) and mechanically (green) activated, normalized for the total duration of the electrical or mechanical activation (as displayed by the red and green bars in the middle panels of Figure 1, respectively). In this representation, a perfect equality of the sequence of electrical and mechanical activation would result in superimposed lines for electrical and mechanical activation. The electrical curve shows a relatively constant slope, indicating a steady progression of the activation along the endocardium. Conversely, the mechanical curve shows a more concave shape and 
positioned significantly $(P<0.01)$ below the electrical curve. The distribution of the mechanical activation curve was skewed towards a slow early and faster late peak shortening. The mechanical activation curve ran significantly $(P<0.01)$ below the electrical activation curve, especially for the scar group (Table 2 and bottom panels of Figure 2).

The segments where the latest electrical and mechanical activations occurred are displayed in Figure 3. Latest electrical activation was most frequently located in the basal sector of the lateral wall, with a few cases in the inferior wall. Although also most frequently located in the lateral wall, mechanical events covered a broader area, affecting also the anterior and posterior wall in a not negligible number of cases. A late electrical or mechanical activation in the septal region was never observed. There was no significant difference in the location of the region of latest electrical activation, but, the region of latest mechanical activation was more variable between patients in the scar group, with a non-negligible number of events observed both in the anterior and inferior walls, apparently not associated with late depolarization in those sectors (Figure 3 lower panels).

\section{Association between electrical and mechanical events}

The variability in the correlation coefficient of the TD-TPS relation between patients was considerable $[R=0.81(0.62-0.88)$, Figure 4$]$.
Of note, all nine patients exhibiting a poor TD-TPS correlation $(R<0.5)$ had evidence of scar. The exclusion of all measurements performed in regions with LGE-identified scar led only to a slight increase of the correlation coefficient $[R=0.84(0.64-0.93)]$, indicating that scar also influenced EM coupling remote from the scar.

Figure 5 illustrates the location of the areas of latest electrical and mechanical activation (extent $20 \%$ of total endocardial area), their overlap and the relation with respect to scar location, for the patients depicted in Figure 1. In Patient A, both areas were located in the midbasal portion of the infero-lateral wall, with a high degree of overlap (96\%, bottom right) and absence of scar (bottom left). Conversely, in Patient B, latest electrical activation took place mainly in the inferiorlateral wall, while its mechanical counterpart covered the anteriorlateral endocardium, resulting in $8 \%$ overlap. Late gadolinium enhancement evidenced a large scar in all mid-lateral sectors. The cumulative results for the overlap are summarized in Figure 4. The overlap displays a distribution similar to the one found for the correlation [64\% (41-78)], with the majority of cases $(n=50)$ showing an overlap area greater than $50 \%$, but with only three of the cases with overlap below $50 \%$ being non-scar. The wider range of low overlap in scarred hearts was confirmed by the distance between the areas of latest electrical and mechanical activation, which was almost twice as large in scar patients compared to non-scar patients (Table 2).
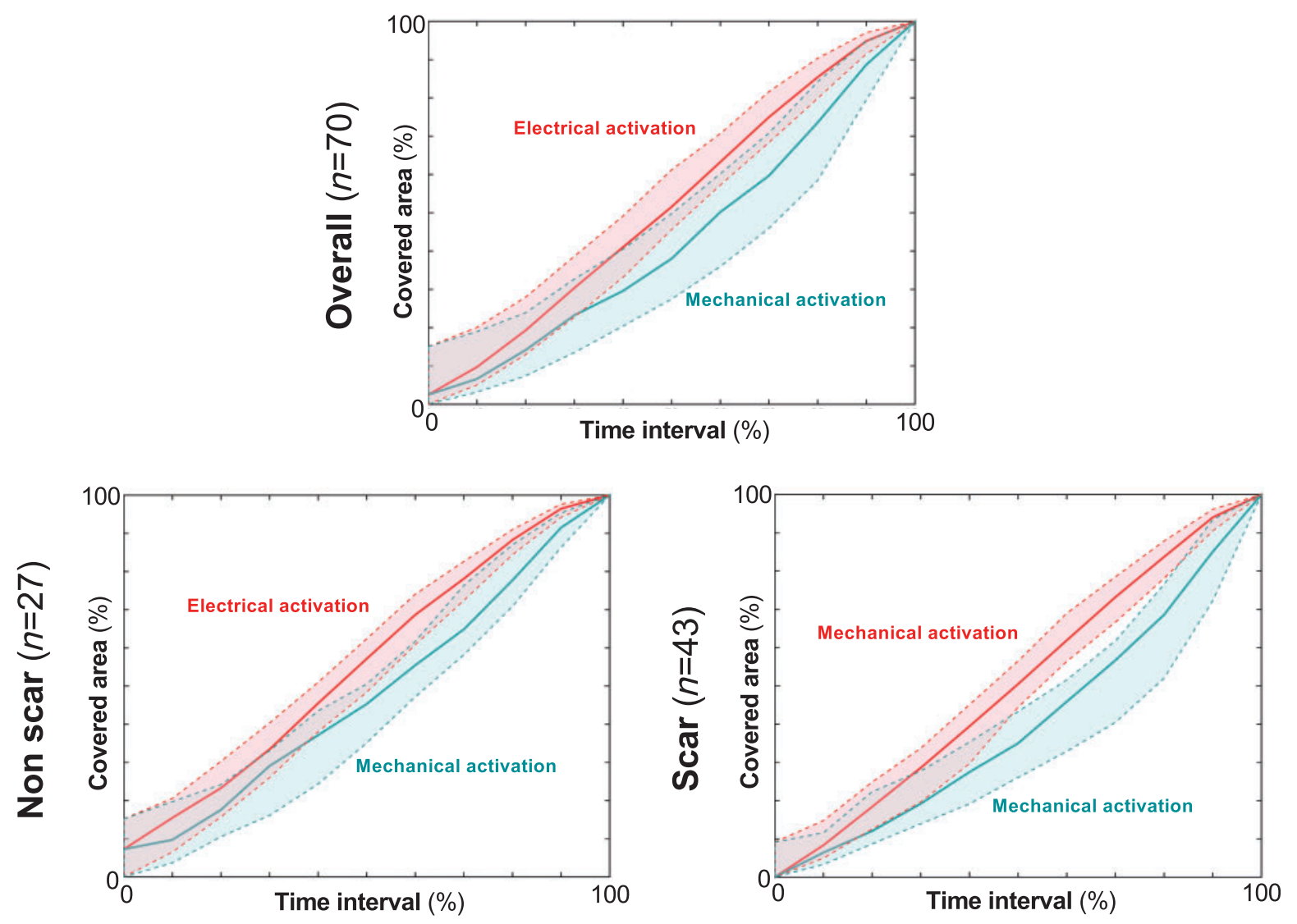

Figure 2 Evolution of electrical (red) and mechanical activation (green) over time, normalized to the total duration of electrical and mechanical activation (see shaded intervals in Figure 1). Presented are median values (thick line) and the area between the 1st and 3rd quartiles (dashed lines) in all 70 patients (upper panel) and separately for the non-scar (bottom panel, left) and scar patients (bottom panel, right). 


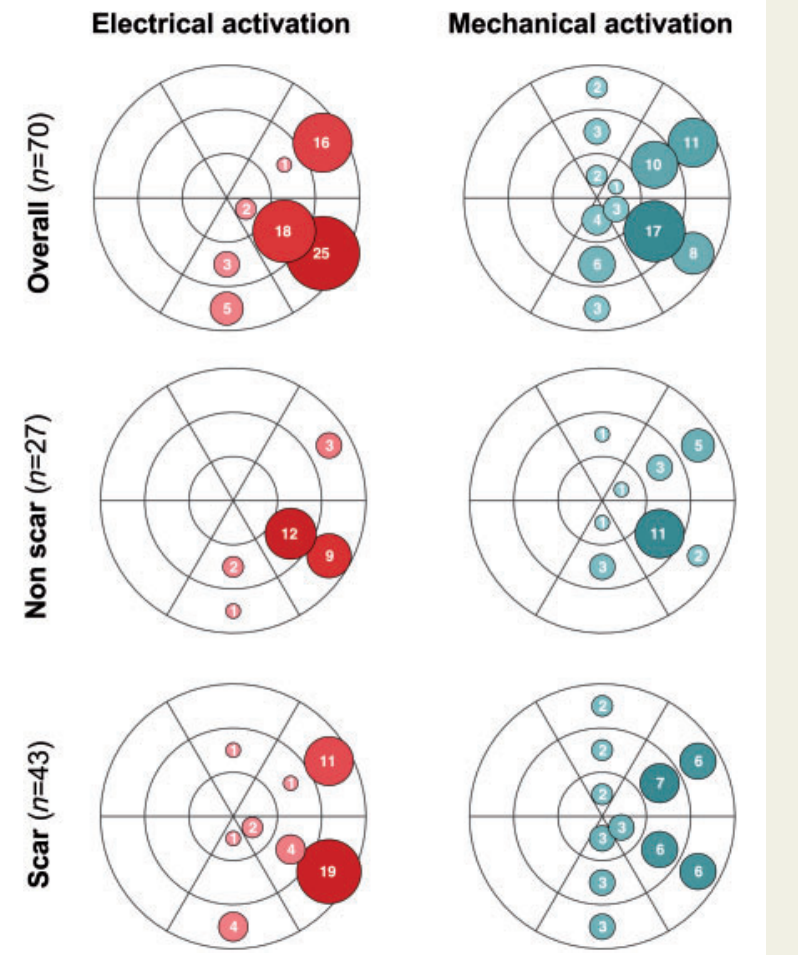

Figure 3 Location of latest activated regions (left) and latest mechanical sites (right), in the overall population (top), and separately for patients with (bottom) and without (mid) evidence of scar.

Despite a significant variability in the association between scar volume and overlap, the proportion of cases with overlap $<60 \%$ was larger in presence of scar, and the location of scar location did not influence the overlap, rather any quantity of scar deteriorates EM coupling (Supplementary material online, Figure S2).

As summarized in Table 3, the use of different thresholds above $50 \%$ of transmurality for the definition of significant scar, did not imply any statistically significant change to the previously described findings. Albeit statistically underpowered, the data in Table 3 do show a trend towards poorer association between electrical and mechanical parameters when using higher (>75\%) transmurality of scar.

Additional analyses were performed to investigate the effects of heart rate (Supplementary material online, Table T1), inclusion bias according to the enrolling centre (Supplementary material online, Table T2) and the aetiology of HF (ischaemic vs. non-ischaemic, Supplementary material online, Table T3). None of these analyses showed a significant effect of the factor used to dichotomize the study group.

\section{Discussion}

To the best of our knowledge, the present study represents the first comprehensive analysis of the in-vivo spatiotemporal relationship between LV electrical and mechanical activation in a large group of HF patients, obtained with an unprecedented precision due to the unique AEMM technique. The main findings of the study are (i) the relation between electrical and mechanical activation is variable but particularly weak in presence of scar; (ii) latest mechanical activation is less likely to occur in the lateral LV wall than latest electrical activation, especially in hearts with scar; (iii) these effects of scar appear independent of the distance between scar and latest activated region, implying that scar also affects EM coupling remotely. Our findings provide guidance for CRT delivery in patients with non-ischaemic cardiomyopathy and even more in patients with ischaemic cardiomyopathy, especially when positioning an endocardial LV pacing lead is considered.

\section{Spatiotemporal aspects of electromechanical coupling}

Unlike previous studies ${ }^{9,10}$ that evaluated the TD-TPS relation by combining speckle tracking echocardiography or MRI and coronary venous electrical mapping in a limited number of points per patient, our measurement was significantly more extensive as it included a few hundred, equally distributed points per LV in 70 patients. Consistent with prior studies using AEMM, we found evidence of a preserved EM coupling in non-scarred hearts as indicated by the significant TD-TPS relation. The presence of scar of considerable size clearly altered EM coupling. While the virtual immobility of the scar is expected to influence late mechanical activation if located in the electrically latest activated region, our results also show that the region of latest mechanical activation is altered when the scar is more remote. After all, the (poor) TD-TPS correlations are based on points all over the LV wall. Moreover, exclusion of all regions with scar from analysis barely improved the TD-TPS correlation.

The effect of scar on EM coupling even in remote regions may be understood from the increased load that a mechanically inactive region imposes on all other regions because together they need to resist the forces imposed by LV pressure. While the higher load will reduce maximal shortening, also timing of peak contraction is different, as non-scarred tissue starts with shortening within tens of milliseconds after depolarization, while the scar tissue is stretched by the rise of LV pressure and only recoils (observed as shortening) when LV pressures drops again.

\section{Association between latest}

\section{depolarization and mechanical activation}

The finding of the majority of electrically latest activated regions in the antero- and infero-lateral regions, mostly in the basal portion, is concordant with previous findings. ${ }^{8,10}$ Based on the 16-segment AHA model, in the present study, the distribution of the electrically and mechanically latest activated regions coincided in $69 \%$ of the cases. This percentage is slightly lower than the $83 \%$ reported by Mafi-Rad et al. ${ }^{10}$ However, in the latter study evidence of scar was only present in three subjects. Similarly, Suever et al. ${ }^{9}$ found correspondence in 10/11 cases (91\%) without prior myocardial infarction. In another study, a match was observed in only $3 / 16$ patients (19\%), but the number of patients with significant scar is not reported. ${ }^{8}$ Therefore, these data from literature suggest that concordance of regions with latest electrical and mechanical activation is related to the percentage of patients with scar. The present study strongly supports and extends this idea, based on more detailed measurements, a larger number of patients and the 


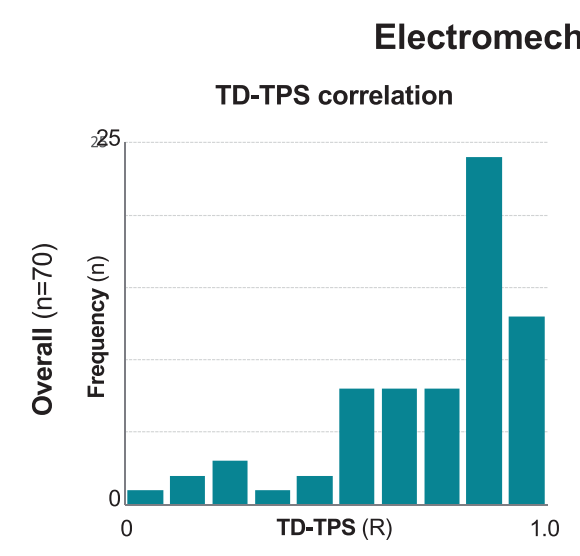

\section{Electromechanical coupling}
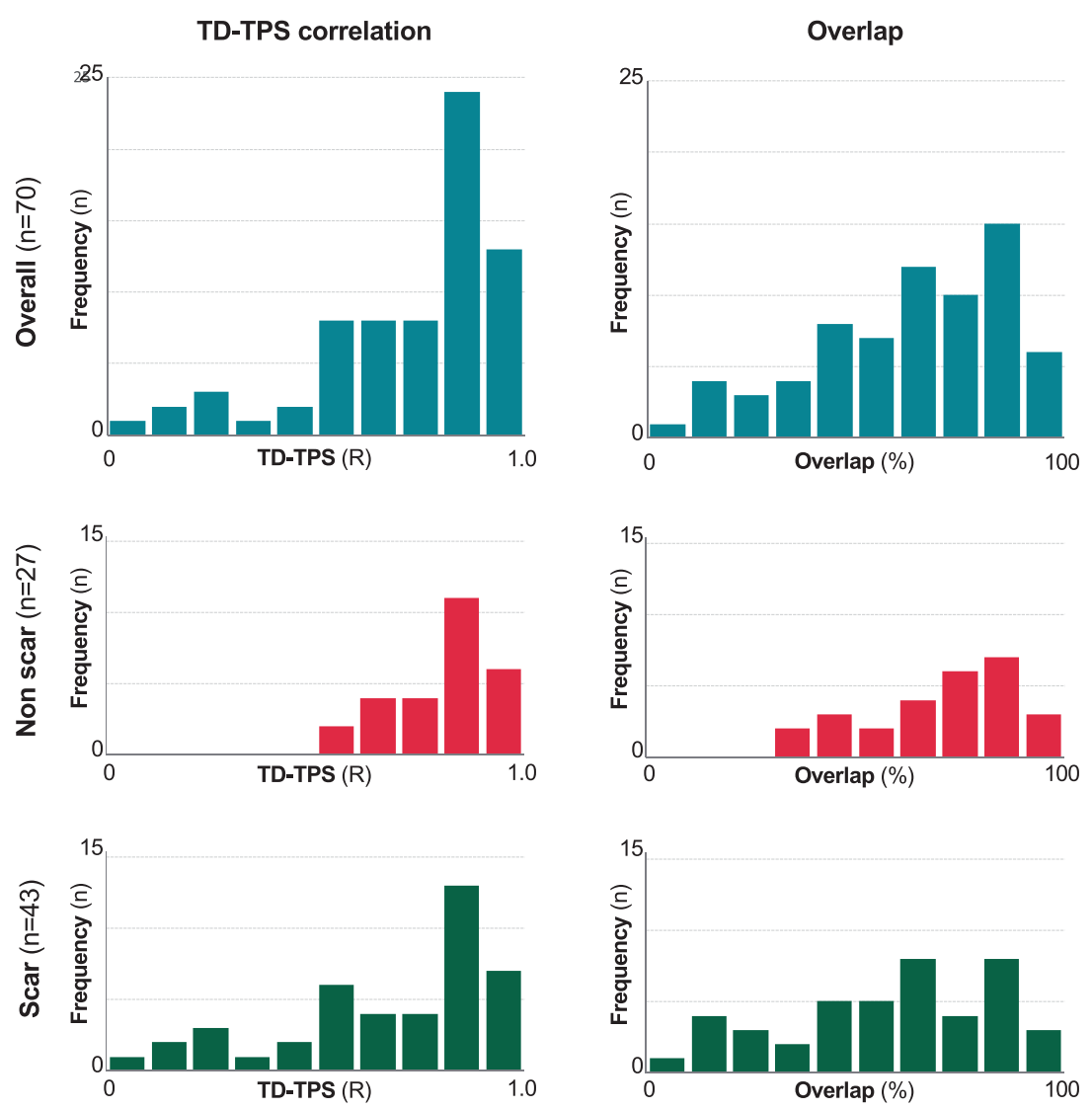

Figure 4 Frequency distribution of the correlation coefficient of the TD-TPS relation (left) and distribution of the overlap between the latest activated mechanical and electrical regions. Distributions are shown for the overall group (top), and separately for patients with (bottom) and without (mid) evidence of scar. TD, time of depolarization; TPS, time-to-peak shortening.

expression of EM coupling by overlap between regions of late electrical and mechanical activation as well as TD-TPS correlation.

\section{Location of late electrical and mechanical activation: implication for left ventricular lead implantation}

The poor overlap between the areas of latest electrical and mechanical activation in scarred hearts has significant implications because both the longitudinal (apex-base) and circumferential (anterior-inferior) position of the LV lead has been implicated in CRT outcome. Conflicting short- and long-term results have been reported for pacing the apical region vs. more basal region., 14 Similarly, experimental evidence and acute LV pacing result in conflict outcome data when comparing anterior, lateral, or posterior LV wall pacing. 1,15,16

Notably, in a subanalysis of the MADIT-CRT trial, an apical LV lead position was associated with a significantly worse clinical outcome compared with non-apical lead positions in LBBB $(P<0.009)$ and non-ischaemic $(P<0.03)$, but not in ischaemic cardiomyopathy $(P=0.16){ }^{2}$ In contrast, Leyva et al. ${ }^{14}$ showed that an apical LV lead position was associated with better long-term cardiac survival than a non-apical position.
Although differences in demographic characteristics and LV pacing location may make a comparison among the different studies challenging, our results provide a unique opportunity for conciliating the controversy. In our study, $65 \%$ of all late activated regions in non-scar patients was located in basal segments. However, when patients without scar were considered, there was a shift towards a more mid-apical position rather than basal position of the late activated area, which is comparable to what has been observed in experimental LBBB in absence of myocardial scar. ${ }^{16}$ While the largest increase in contractility in non-scarred hearts was observed by pacing the mid-apical position, in the presence of scar, this area was smaller, more basally located, and dependent on scar location. ${ }^{17}$

Our data also help in shedding lights in the controversy of circumferential lead positioning. Although latest electrical and mechanical activations were more frequently positioned in the lateral wall, it was in the anterior and inferior part of LV in about $25 \%$ and $11 \%$ of patients, respectively. Scar presence significantly influenced the distribution of latest activations, as non-laterally located area of latest activations, both electrical and mechanical, were more frequent in patients with scar. Different from the LV lead optimization at longitudinal position, where exploration is facilitated by the availability of 

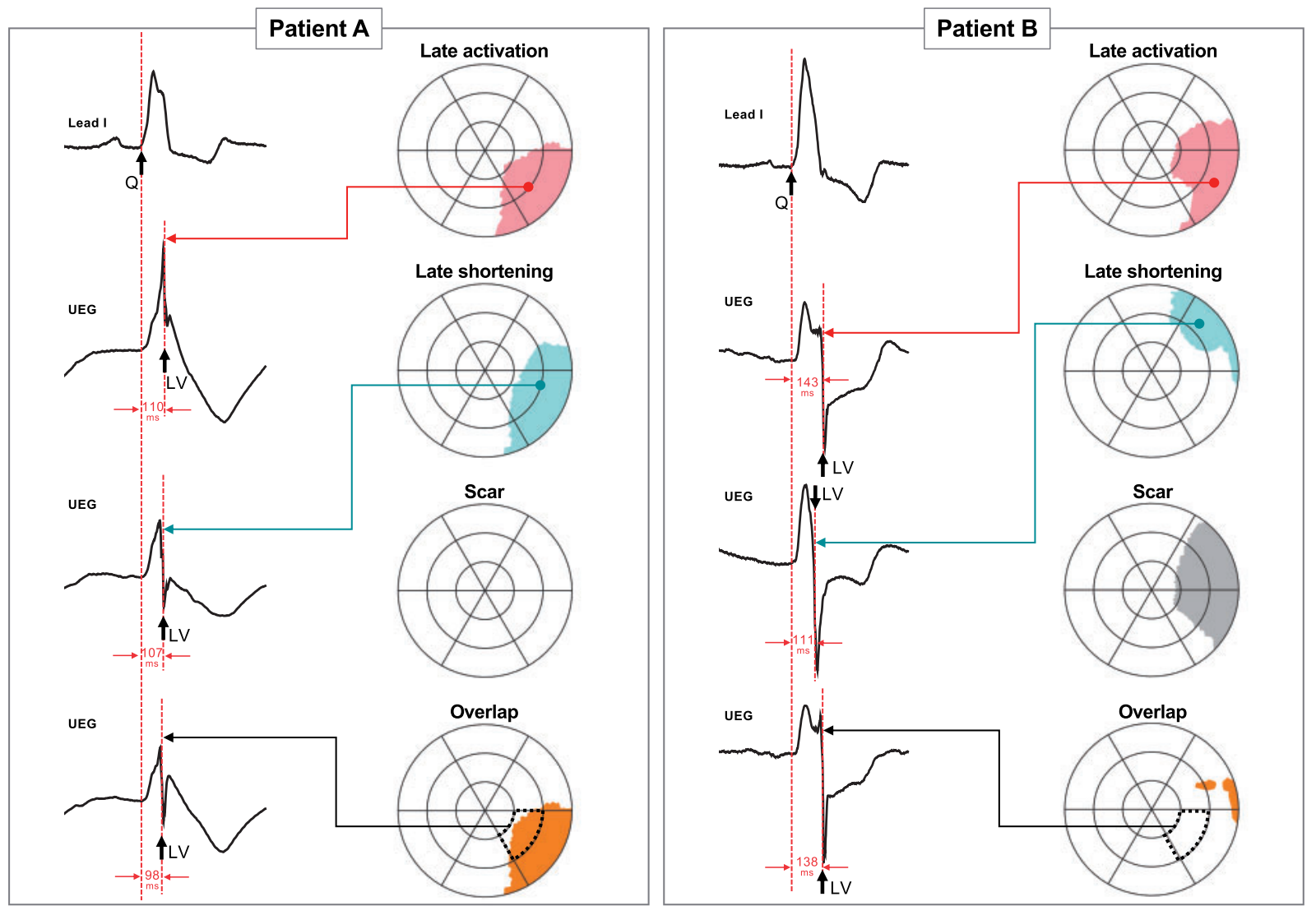

Figure 5 Examples of the spatial association between the $20 \%$ area of latest electrical and mechanical activation, their overlap and the local scar burden, in the same patients shown in Figure 1. The unipolar electrograms measured in correspondence of the latest electrical region, latest mechanical region, and over the mid inferolateral sector are shown on the let with respect with the ECG signal (Lead I). ECG, electrocardiogram.

\section{Table 3 Effects of the scar transmurality threshold on parameters of EM coupling}

\begin{tabular}{|c|c|c|c|}
\hline & $\begin{array}{l}\text { Transmurality } 50-75 \% \\
\text { ( } n=18 \text { patients) }\end{array}$ & $\begin{array}{l}\text { Transmurality }>75 \% \\
(n=25)\end{array}$ & P-value \\
\hline Number of endocardial sites & $234(201-268)$ & $229(190-264)$ & 0.21 \\
\hline TD-TPS correlation $(R)$ & $0.80(0.55-0.88)$ & $0.76(0.57-0.87)$ & 0.67 \\
\hline Area between $E$ and $M$ curves over time & $11.6(8.1-17.9)$ & $14.8(7.919 .2)$ & 0.84 \\
\hline Distance between electrical and mechanical centroids (\%) & $14.5(7.5-27.8)$ & $21.7(8.5-30.6)$ & 0.72 \\
\hline Overlap (\%) & $64(38-77)$ & $55(32-72)$ & 0.61 \\
\hline
\end{tabular}

Data are expressed as median (1st-3rd) quartiles. P-value refers to the transmurality $50-75 \%$ vs. transmurality $>75 \%$ comparison and Mann-Whitney $U$ test.

EM, electromechanical; TD, time of depolarization; TPS, time-to-peak shortening.

quadripolar leads, individualization of a circumferential position is significantly more challenging as it requires an acute electrical and hemodynamic evaluation for each available vein. ${ }^{15,18}$ However, when this is done, sites with longest Q-LV interval yield the largest increase in LV contractility ${ }^{18}$ thus, confirming that an electrically late activated area is worth finding. Finally, the endocardial measurements performed in this study will be particularly applicable to the placement of $L V$ endocardial pacing leads.

\section{Clinical implications for cardiac imaging selection}

Until now, the use of mechanical dyssynchrony for lead positioning is advocated because of the non-invasiveness of echocardiography and CMR. ${ }^{19}$ The present study shows that the presence of scar disconnects electrical and mechanical dyssynchrony. This finding may seem at odds with the positive results of the STARTER and TARGET trials that used mechanical dyssynchrony to target the positioning of the 
LV lead. ${ }^{5,20}$ In this respect it should be noted that the present study did not include the measurement of any outcome. However, it is also important to note that in the clinical trial regions with scar (as indicated by low strain amplitude, thin LV wall) were excluded and that the benefit of excluding such regions was almost as large as that of implantation at the latest activated region.

Furthermore, when comparing the EM measurements between patients with ischaemic and non-ischaemic HF, we could not find any significant difference. This further suggests that the presence of significant scar and not the aetiology of HF plays a major role in disconnecting the electrical and mechanical dyssynchrony.

Our results may be of interest to not only implanters of CRT devices but also to a broad part of the cardiology community, including those involved in the management of HF patients, intraprocedural imaging integration, optimization of target delivery of biological therapy, and planning a prospective randomized controlled trial for individualizing CRT.

In the light of the results of this study, LV lead positioning should not be based solely on measuring mechanical dyssynchrony, rather in conjunction with the evaluation of the pattern of electrical activation. Invasive (Q-LV, RV-LV interval, and ECG imaging) seems more useful, especially when combined with scar imaging in order to avoid positioning of the lead in a scarred region. ${ }^{13}$ The growing availability and adoption of ECG-imaging technologies may facilitate the accurate identification of the site of latest electrical activation. However, the benefit of these non-invasive electrical approaches needs to be supported by future trials.

\section{Limitations}

Mechanical activation has been determined as the time of peak shortening. While the onset of shortening is closer to the time of depolarization, its determination is more challenging and more sensitive to noise.

Effects of the scar transmurality threshold on parameters of EM coupling have been evaluated in small groups, resulting in underpowered comparisons. However, this does not affect the fact that even remote scar could affect EM coupling.

In clinical practice, LV pacing is commonly delivered at epicardial site thus, one may question whether our measurements are clinically relevant. However, transmural time, i.e. the activation time from endocardium to epicardium, is usually very short and ranges between 10 and $15 \mathrm{~ms}^{16}$; furthermore, there is a high correlation between catheter-based local strain with strain measured by cardiac resonance imaging. ${ }^{12}$

\section{Conclusions}

Our study indicates that the relation between electrical and mechanical activation is variable and particularly weak in hearts with scar. Importantly, the effects of scar appeared independent of the distance between scar and latest activated region, implying that scar also affects EM coupling remote from the scar. Our findings provide guidance for CRT delivery in patients with non-ischaemic cardiomyopathy and even more in patients with ischaemic cardiomyopathy, especially when positioning of an LV endocardial pacing is considered. Albeit the study was not designed to compare LV lead positioning based on electrical vs. mechanical activation, our results suggest that LV lead positioning should not be guided solely by measurements of mechanical dyssynchrony, as mechanical and electrical activation patterns might not overlap perfectly, especially when significant scar is observed.

\section{Supplementary material}

Supplementary material is available at Europace online.

Conflict of interest: F.M. reports intellectual property with Biosense Webster. A.A. is a consultant to Boston Scientific, Backbeat, Biosense Webster, Cardiac, Corvia, Daiichi-Sankyo, Medtronic, Merit, Microport CRM, Philips, and V-Wave; he received speakers' fee from Daiichi-Sankyo, Boston Scientific, Biosense Webster, Medtronic, Microport CRM, and Philips; he participated in clinical trials sponsored by Boston Scientific, Medtronic, Microport CRM, and Zoll Medical; he reports intellectual properties with Boston Scientific, Biosense Webster, and Microport CRM. F.R. is a consultant for Daiichi-Sankyo, Bayer, and Boston Scientific. He received speaker fees from Medtronic and Microport. F.P. has received research grants from Medtronic, Abbott, Microport CRM, Biotronik, and Biosense Webster and speakers fee from Medtronic, Abbott, and Microport CRM. He reports intellectual property with Boston Scientific, Medtronic, and Biosense Webster. K.S.G. received speakers' fee from Medtronic and participated in clinical trials sponsored by Medtronic and Abbott. All other authors have no conflict of interest to declare.

\section{Funding}

This work was in part supported by grants from the Swiss National Science Foundation under project 32003B_165802, from the Swiss Heart Foundation, and by a restricted grant of Biologic Delivery Systems, Division of Biosense Webster a Johnson \& Johnson Company. Statutory funds of the Medical University of Silesia [KNW-1-135/N/6/K] and [POIG.01.01.02-00-109/09] grant to W.W.

\section{References}

1. Bristow MR, Saxon LA, Boehmer J, Krueger S, Kass DA, De Marco T et al. Cardiac-resynchronization therapy with or without an implantable defibrillator in advanced chronic heart failure. N Engl ] Med 2004;350:2140-50.

2. Singh JP, Klein HU, Huang DT, Reek S, Kuniss M, Quesada A et al. Left ventricular lead position and clinical outcome in the multicenter automatic defibrillator implantation trial-cardiac resynchronization therapy (MADIT-CRT) trial. Circulation 2011;123:1159-66.

3. Tang ASL, Wells GA, Talajic M, Arnold MO, Sheldon R, Connolly S et al. Cardiac-resynchronization therapy for mild-to-moderate heart failure. $N$ Engl J Med 2010;363:2385-95.

4. Cleland JGF, Daubert JC, Erdmann E, Freemantle N, Gras D, Kappenberger L et al. The effect of cardiac resynchronization on morbidity and mortality in heart failure. N Engl J Med 2005;352:1539-49.

5. Khan FZ, Virdee MS, Palmer CR, Pugh PJ, O'Halloran D, Elsik M et al. Targeted left ventricular lead placement to guide cardiac resynchronization therapy: the TARGET study: a randomized, controlled trial. J Am Coll Cardiol 2012;59: 1509-18.

6. Gold MR, Birgersdotter-Green U, Singh JP, Ellenbogen KA, Yu Y, Meyer TE et al. The relationship between ventricular electrical delay and left ventricular remodelling with cardiac resynchronization therapy. Eur Heart J 2011;32:2516-24.

7. Prinzen FW, Augustijn CH, Allessie MA, Arts T, Delhass T, Reneman RS. The time sequence of electrical and mechanical activation during spontaneous beating and ectopic stimulation. Eur Heart J 1992;13:535-43.

8. Fujiwara R, Yoshida A, Fukuzawa K, Takei A, Kiuchi K, Itoh M et al. Discrepancy between electrical and mechanical dyssynchrony in patients with heart failure and an electrical disturbance. Pacing Clin Electrophysiol 2014;37:576-84. 
9. Suever JD, Hartlage GR, Magrath RP, Iravanian S, Lloyd MS, Oshinski JN. Relationship between mechanical dyssynchrony and intra-operative electrical delay times in patients undergoing cardiac resynchronization therapy. J Cardiovasc Magn Reson 2014;16:4.

10. Mafi-Rad M, van'T Sant J, Blaauw Y, Doevendans PA, Cramer MJ, Crijns HJ et al. Regional left ventricular electrical activation and peak contraction are closely related in candidates for cardiac resynchronization therapy. JACC Clin Electrophysiol 2017;3:854-62.

11. Kroon W, Lumens J, Potse M, Suerder D, Klersy C, Regoli F et al. In-vivo electromechanical assessment of heart failure patients with prolonged QRS duration. Heart Rhythm 2015;12:1259-67.

12. Maffessanti F, Prinzen FW, Conte G, Regoli F, Caputo ML, Suerder D et al. Integrated assessment of left ventricular electrical activation and myocardial strain mapping in heart failure patients: a holistic diagnostic approach for endocardial cardiac resynchronization therapy, ablation of ventricular tachycardia, and biological. JACC Clin Electrophysiol 2018;4:138-46.

13. Nguyen UC, Potse M, Vernooy K, Mafi-Rad M, Heijman J, Caputo ML et al. A left bundle branch block activation sequence and ventricular pacing influence voltage amplitudes: an in vivo and in silico study. Europace 2018;20(Suppl_3):iii77-86.

14. Leyva F, Zegard A, Taylor RJ, Foley PWX, Umar F, Patel K et al. Long-term outcomes of cardiac resynchronization therapy using apical versus nonapical left ventricular pacing. I Am Heart Assoc 2018;7:1-13.

15. Auricchio A, Stellbrink C, Butter C, Sack S, Vogt J, Misier AR et al. Clinical efficacy of cardiac resynchronization therapy using left ventricular pacing in heart failure patients stratified by severity of ventricular conduction delay. J Am Coll Cardiol 2003;42:2109-16.

16. Strik M, Rademakers LM, van Deursen CJM, van Hunnik A, Kuiper M, Klersy C et al. Endocardial left ventricular pacing improves cardiac resynchronization therapy in chronic asynchronous infarction and heart failure models. Circ Arrhythm Electrophysiol 2012;5:191-200.

17. Rademakers LM, van Kerckhoven R, van Deursen CJM, Strik M, van Hunnik A Kuiper $M$ et al. Myocardial infarction does not preclude electrical and hemodynamic benefits of cardiac resynchronization therapy in dyssynchronous canine hearts. Circ Arrhythm Electrophysiol 2010;3:361-8.

18. Zanon F, Baracca E, Pastore G, Marcantoni L, Fraccaro C, Lanza D et al. Multipoint pacing by a left ventricular quadripolar lead improves the acute hemodynamic response to CRT compared with conventional biventricular pacing at any site. Heart Rhythm 2015;12:975-81.

19. Behar JM, Jackson T, Hyde E, Claridge S, Gill J, Bostock J et al. Optimized left ventricular endocardial stimulation is superior to optimized epicardial stimulation in ischemic patients with poor response to cardiac resynchronization therapy: a combined magnetic resonance imaging, electroanatomic contact mapping, and hemodynamic study to target endocardial lead placement. JACC Clin Electrophysiol 2016:2:799-809.

20. Adelstein E, Alam MB, Schwartzman D, Jain S, Marek J, Gorcsan J et al. Effect of echocardiography-guided left ventricular lead placement for cardiac resynchronization therapy on mortality and risk of defibrillator therapy for ventricular arrhythmias in heart failure patients. Am J Cardiol 2014;113:1518-22.

\section{Corrigendum}

doi:10.1093/europace/euaa033

Online publish-ahead-of-print 30 March 2020

Corrigendum to: Maffessanti F, et al. The influence of scar on the spatio-temporal relationship between electrical and mechanical activation in heart failure patients [Europace 2020;22:777-786]

In an earlier version of this manuscript Radosław Kurzelowski's affiliation was incorrectly identified. This has now been corrected to 'Division of Cardiology and Structural Heart Diseases, Medical University of Silesia, Katowice, Poland'. In addition, Tomasz Jadczyk has had an additional affiliation added: 'Interventional Cardiac Electrophysiology Group, International Clinical Research Center, St. Anne's University Hospital Brno, Czech Republic', and Magdalena Dą browska’s first name has been corrected. 